\title{
Immediate implant placement and provisionalization following tumor-resective surgery in the midfacial region: A case series
}

Nelson Fernandes, BDS ${ }^{a,{ }^{*}}$, Jacobus van den Heever, BChD, MChD (Pros) ${ }^{b}$, Leanne Sykes, BSc, BDS, MDent (Pros) ${ }^{\mathrm{c}}$.

\footnotetext{
${ }^{a}$ Resident registrar, Department of Prosthodontics, School of Dentistry, Faculty of Health Sciences, University of Pretoria, South Africa.

${ }^{b}$ Consultant prosthodontist, Department of Prosthodontics, School of Dentistry, Faculty of Health Sciences, University of Pretoria, South Africa.

${ }^{c}$ Associate professor and head of clinical unit, Department of Prosthodontics, School of Dentistry, Faculty of Health Sciences, University of Pretoria, South Africa.
}

*Address for correspondence: Dr. NA Fernandes, Department of Prosthodontics, PO Box 1266, Pretoria, 0001, South Africa.

Tel: +27123192681; Fax: +27123230561; e-mail: nelsondentist@gmail.com Disclosure: No conflict of interest declared.

\section{Abstract}

Surgical and prosthodontic restoration of the midfacial region following tumor resection has always posed a considerable challenge, as this area serves crucial functional and esthetic roles. Being diagnosed and subsequently treated for facial tumors can have an immense psychosocial impact on a patient, as the resulting defects are often disfiguring, and lead to an inability to masticate, swallow, and speak clearly. Provision of an immediate facial and dental prostheses at the time of surgery can limit these side effects and help reduce mental duress on these patients 
and their families, as well as aid in the process of rehabilitation. Rapid prototyping (RP) and three-dimensional (3D) printing, as this paper shows, assists pre-surgical planning of the tumor resection, as well as the manufacture of maxillo-facial and dental prostheses. Oftentimes these defects are extensive, so prostheses retention is aided by zygomatic implants which are placed at the time of surgical resection. When placed at this time, and prior to radiation therapy, these craniofacial implants have improved survival rates. Thus, this treatment modality can improve postoperative recovery considerably, while at the same time allowing for cleaning and monitoring of the resected site for tumor recurrence.

Keywords: obturators; facial prostheses; zygomatic implants

\section{Introduction}

Surgical midfacial reconstruction following tumor ablation is challenging, as it attempts to achieve many objectives. These include: supporting the orbital contents; cosmetic improvement of the exenterated orbit; maintenance of a patent nasal airway with oronasal separation; provision of a stable oral platform for mastication and speech; and recreation of symmetrical contours of the reconstructed site with that of the contralateral face. ${ }^{1}$ While advancements in head and neck surgery, via the use of microvascular techniques and free tissue transfers, have enabled surgeons to reconstruct missing structures and achieve many of these goals, this is not always possible in the midfacial region. ${ }^{2}$ Currently, no single reconstructive technique is able to achieve all these goals, thus a multidisciplinary approach is advocated. $^{3}$ 
The incorporation of prosthetic appliances in these reconstructions has been shown to provide excellent results. ${ }^{3}$ The fabricated facial prostheses can replace large sections of hard and soft tissues, and can be sculpted to mimic anatomical contours and details while simultaneously avoiding the tissue distortion often associated with surgical reconstruction. Being removable, they also allow for cleaning and regular surveillance of the tumor resection site. ${ }^{4,5}$

Extraoral implants have been used successfully for many years for prostheses fixation in the auricular, orbital, and nasal regions. ${ }^{4}$ In patients with extensive midfacial defects, combining craniofacial prostheses with intraoral dentures allows for near complete restoration of both cosmetic and functional requirements. This is also less invasive, as it eliminates the need for further surgical reconstruction. ${ }^{6}$ Zygomatic implants were originally developed for restoring extensively resorbed posterior maxillae without the need for further bone augmentation. ${ }^{7}$ In severe defects, resulting from partial or complete maxillectomies, it has been found that these implants can also provide adequate retention for craniofacial prostheses. ${ }^{8}$ They allow full arch reconstructions in severe defects, and can also be used to support extraoral nasal prostheses in cases with oronasal communication. ${ }^{6}$ Utilizing prosthetic devices to reconstruct maxillary defects following tumor resection allows for visual examination of the resected site for signs of possible superficial recurrence. Though imaging modalities have improved significantly for monitoring such recurrence, early visual detection could possibly prevent further aggressive surgery. This can only be achieved prosthetically, therefore, current literature still supports the need for extraoral prosthetic devices to rehabilitate large midfacial defects. $^{6,8-13}$ 
This paper discusses four patients who were all left with extensive maxillary defects following tumor resection. All four had zygomatic implants immediately placed at the time of tumor resection surgery. These implants aided in the immediate provisionalization of these patients, as they retained and supported craniofacial prostheses and obturators/intraoral dentures.

\section{Case report}

Four patients, all requiring orofacial rehabilitation following extensive midfacial tumor resective surgery involving most of their maxillae (subtotal maxillectomy), are discussed. The resulting defects all needed some form of facial and oro-dental prostheses for rehabilitation. The aggressive nature of these tumors also necessitated some patients to undergo post-operative radiation or chemotherapy. Zygomatic implant positioning was discussed during the pre-surgical planning phase for each patient, and marked on the three-dimensional (3D) stereolithographic model obtained for each patient. This gave the surgeons an approximate indication of implant angulation during insertion and the depth of placement. The implants were placed at the time of tumor resection in all patients, and these patients were immediately provisionalized with interim dentures and facial prostheses where necessary. Regular recall appointments were scheduled for all patients to monitor their progress, and adjustments were made to their devices in accordance with the tissue changes encountered during healing. Definitive prostheses were manufactured after adequate recovery had taken place (within the following 3-6 months). 


\section{Case 1}

A 77-year-old male patient presented with a swelling in his right paranasal sinus region. Medical history revealed that he had undergone tumor resection for a basal cell carcinoma of this area 10 years previously. Recurrences had since occurred on two separate occasions. In both instances, the tumors had been removed and the defective site surgically reconstructed with local flaps followed by a course of radiotherapy.

At the time of presentation, his third tumor recurrence, radiological and histological findings revealed that it had spread widely, resulting in destructive infiltration of the palate, upper lip, and nasal cavity. Clinically, the patient's anterior maxillary teeth were mobile, and he had a bloody discharge from his nose, and reported to be experiencing blockage of both nasal passages.

Computed tomography (CT) scans were taken of the patient to assess tumor extension. DICOM (digital communication in medicine) files from these scans were transferred into a computer software program (Mimics; Materialise NV) for 3D data processing. Once processed, this data was converted into standard tessellation/triangulation language (.stl) file format, which is the standard used for rapid prototyping and 3D model printing. A 3D model of the patient's maxilla and associated structures was then manufactured, and used for pre-surgical planning. Together with the surgical team, this model was used to plan the extent of the resective surgery, and the positioning of zygomatic implants. Once consensus was reached on this, the model was trimmed accordingly (model surgery), and a 
duplicate plaster cast was manufactured. This cast was then used to fabricate a partial denture, which would be fitted at the time of resective surgery.

The patient's skin tone was acquired with a spectrophotometer (Quickweigh; Spectromatch Ltd.), and a 3D facial scan was taken of the patient's face preoperatively, using a laser scanner (Vivid 910; Konica Minolta Inc.). The proposed defect was sculpted on specialized software (Freeform Modelling V12, Geomagic; 3D Systems Corp.) (Fig 1), after which another 3D model was manufactured. From this model, a wax-replica of the nose was made, and an interim nasal prosthesis was manufactured from silicone (Fig 2).

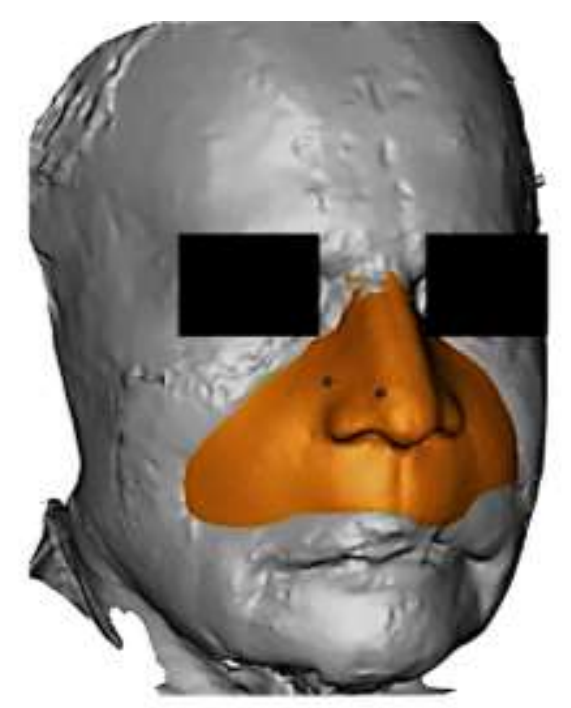

Figure 1 Pre-operative 3D facial scan. 


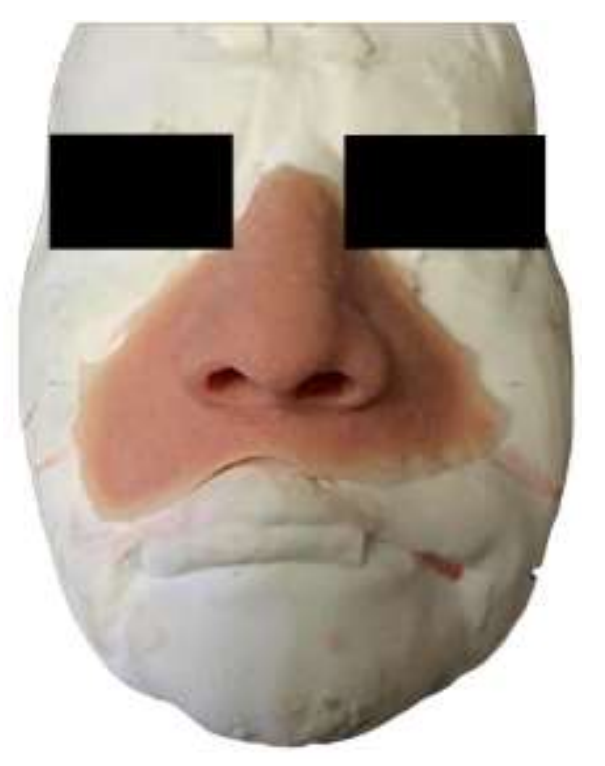

Figure 2 Interim silicone nasal prosthesis on duplicate cast of patient's face.

Surgery involved resection of the entire maxilla anterior to the upper second molars en bloc, including the nose, nasal cavity, and parts of the cheek. Once completed, two zygomatic implants were immediately placed into the zygomatic buttress on either side, as these are areas of good bone density. Titanium cylinders were then attached to these implants, and the dental prosthesis was altered and luted to these cylinders with a self-curing, soft denture relining material (Ufi Gel SC; Voco GmbH) (Fig 3). Titanium screws were then used to retain the dental prosthesis (obturator), and the interim facial prosthesis was fitted and retained by a central magnet attached to the dental prosthesis (Fig 4). 


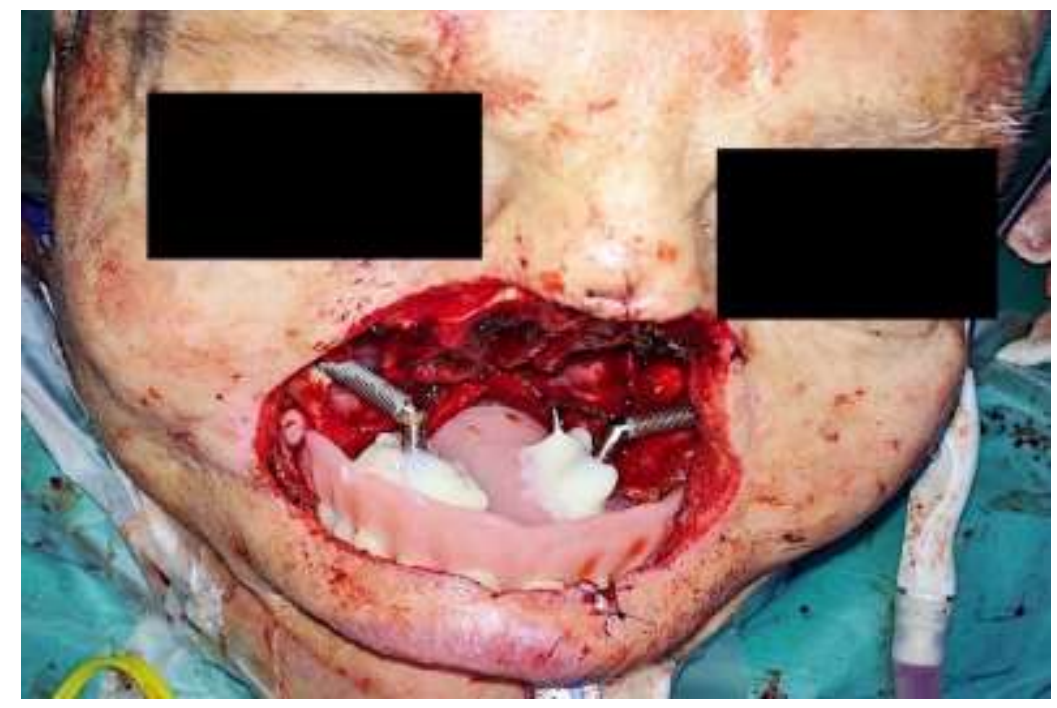

Figure 3 Extensive maxillary resection with dental prosthesis luted onto zygomatic implants.

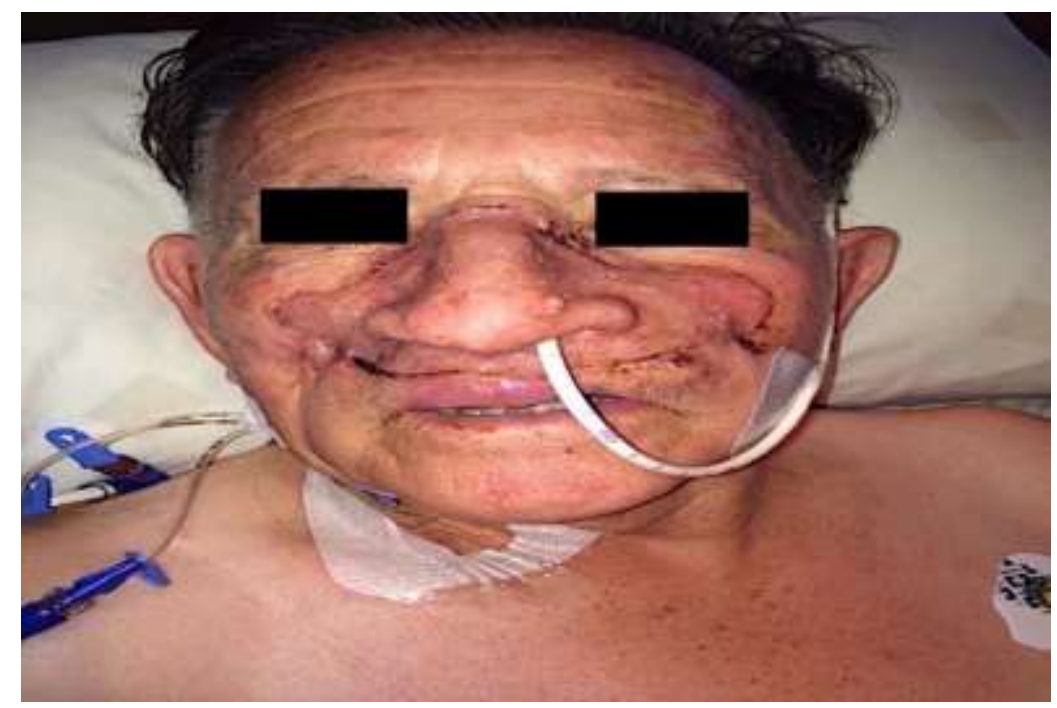

Figure 4 Frontal view of the patient with interim facial prosthesis.

The patient underwent an uneventful recovery, and was followed-up monthly for three months. Final dental and facial prostheses will be manufactured and fitted after six months. 


\section{Case 2}

A 66-year-old male patient presented with a large maxillary lesion that had been present for over three years. CT scans revealed an extensive radiolucent mass that had obliterated the entire right maxillary antrum, with erosion crossing the midline and extending into the nasal cavity. Incisional biopsy and histological examination of the lesion diagnosed this as an ameloblastoma.

The scans revealed that the tumor extended from the right maxillary third molar to the left maxillary bicuspid. Pre-surgical planning was done on a 3D printed model of the patient's maxilla, which was obtained in a similar manner to that described in the previous case. Following model surgery, and duplicate cast manufacture, an interim clear acrylic obturator was then manufactured prior to surgery. Complete surgical excision of the tumor was obtained through bilateral subtotal maxillectomies, a process whereby the orbital floors are left intact. ${ }^{14}$ Three oncology implants were then inserted, two in the right zygoma and one horizontally in the left maxilla (Fig 5).

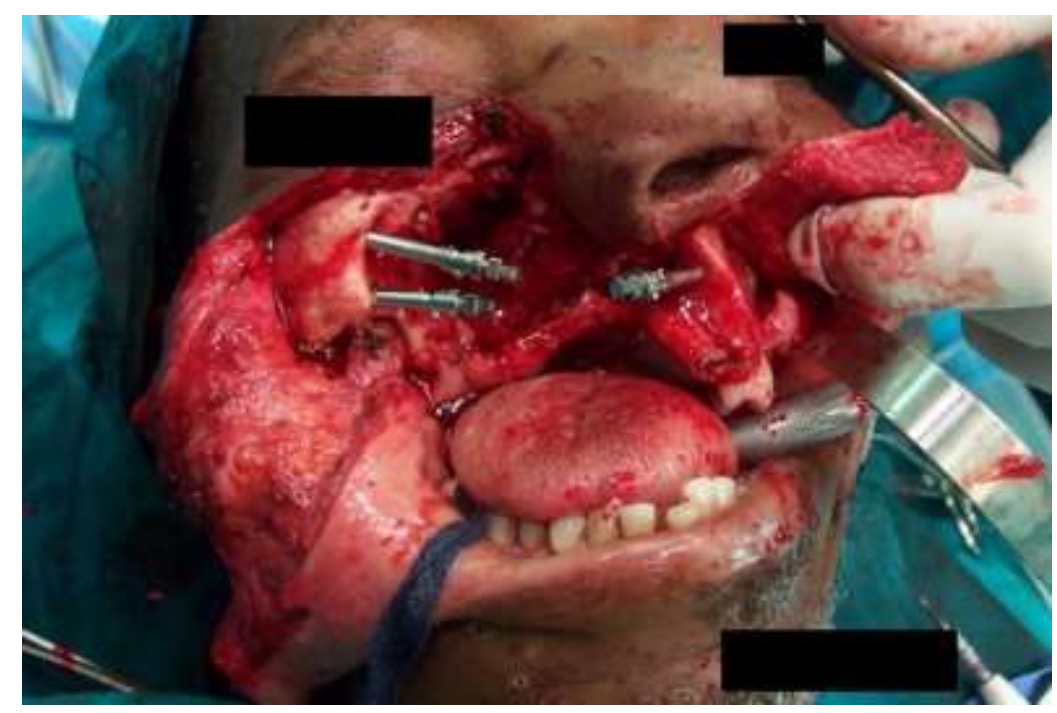

Figure 5 Surgical right subtotal maxillectomy with two oncology implants in the right zygoma and one oncology implant in the left maxilla. 
Zygomatic oncology implants are slightly modified zygomatic implants that have a smooth, non-threaded machined surface along the coronal aspect of their bodies. This produces a maintainable surface that allows for soft tissue integration and prosthesis retention. ${ }^{8}$ Titanium cylinders were attached to the implants using titanium screws, and perforations were made in the obturator in the areas where it was to fit over the cylinders once in position. The obturator was luted to these cylinders intraoperatively by means of a fast setting acrylic resin material (Protemp; 3M ESPE).

Once the material had set, the titanium cylinders were loosened from the implants, and the obturator was trimmed and polished. The defect was then packed with sterile bismuth iodine paraffin paste (BIPP) impregnated gauze, and the obturator was fixed into position onto the implants using titanium screws (Fig 6). BIPP impregnated gauze is commonly used following maxillectomies, as it seals defects, and has excellent astringent and antiseptic properties, which aid post-operative healing. ${ }^{15}$

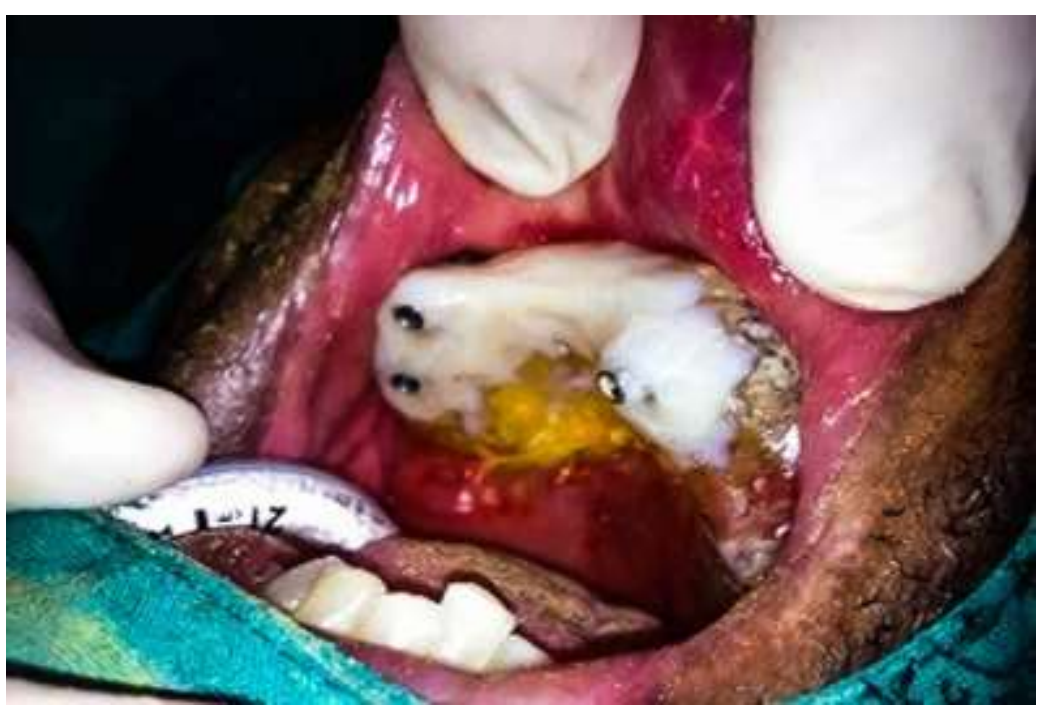

Figure 6 Interim clear acrylic obturator attached to implants by means of titanium cylinders and screws. 
Following three months of uneventful healing, an implant-supported titanium milled bar with locator attachments was manufactured and fitted, to support and retain an overdenture/obturator prosthesis (Figs 7 and 8).

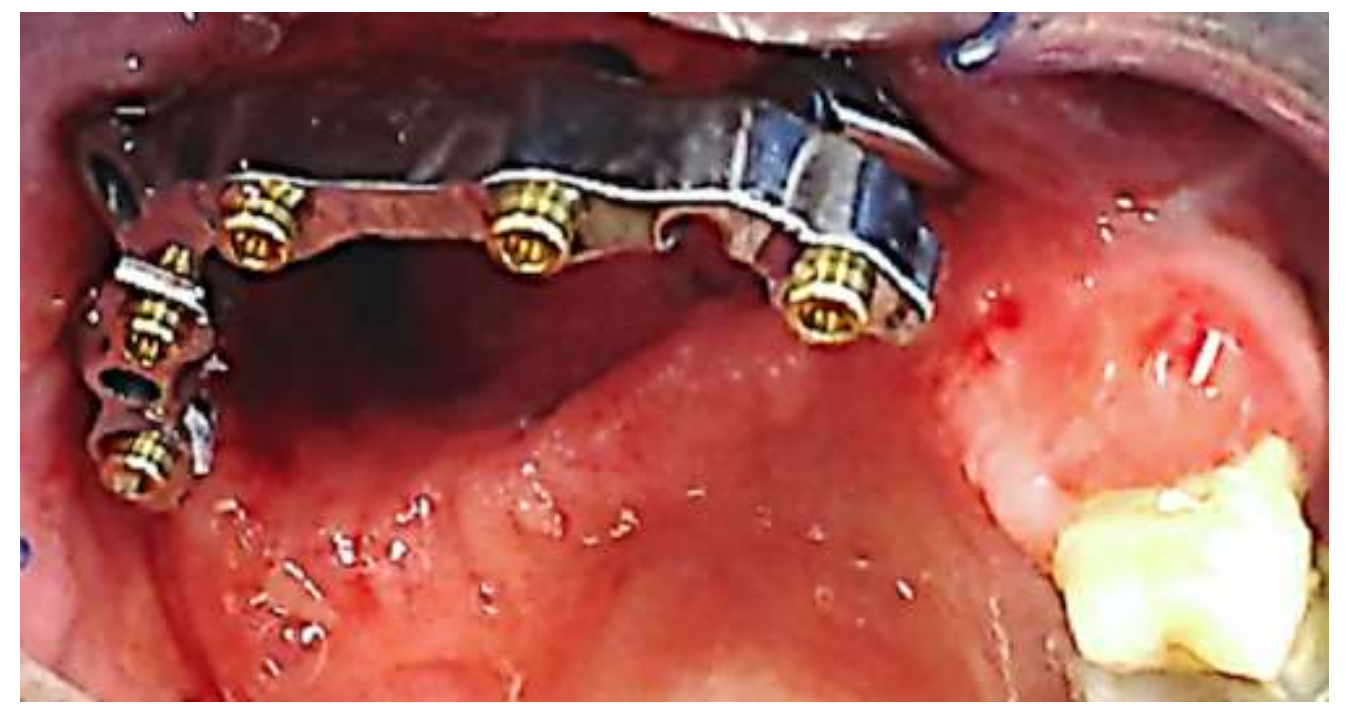

Figure 7 Intra-oral view of implant-supported titanium milled bar with locator attachments.

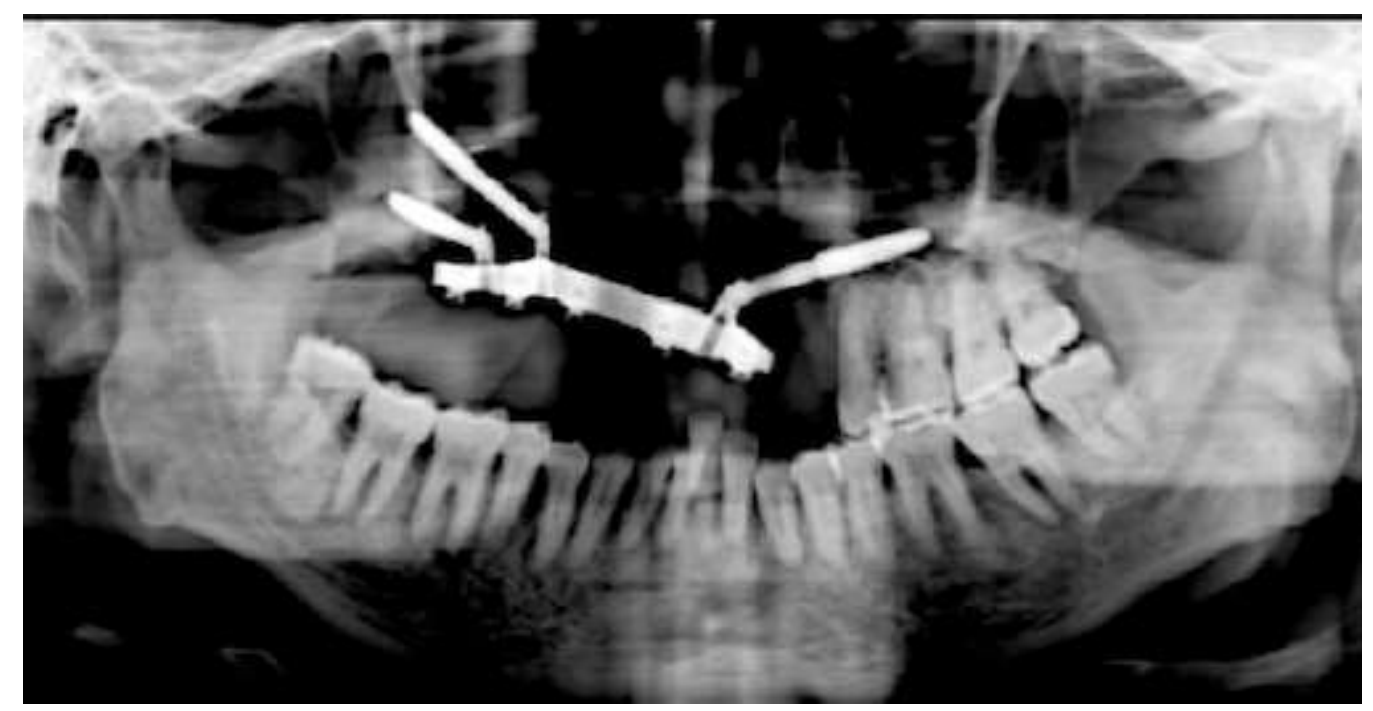

Figure 8 Panoramic radiographic view of implant-supported titanium milled bar. 


\section{Case 3}

A 44-year-old male patient consulted a dentist for pain in his upper right lateral incisor. An endodontic treatment was carried out on this tooth, as the pain was thought to have been of endodontic origin, but the pain never subsided. Upon clinical re-examination, an abnormal mass was noted on the buccal mucosa in this region. The patient was then referred to a maxillo-facial and oral surgeon, who performed an incisional biopsy of the mass, which was then diagnosed as a chondroblastic osteosarcoma upon histological examination.

CT scans showed extensive tumor infiltration into the right maxillary antrum. Chemotherapy was initiated to reduce the size of the mass, so that complete tumor excision could be accomplished with surgery. Once chemotherapy was complete, new CT scans were taken and 3D models were manufactured for pre-surgical planning. A dental prosthesis and obturator were then constructed prior to tumor excision.

Tumor removal involved a subtotal maxillectomy of the right maxilla, followed by the placement of two zygomatic implants into the right zygoma. The right orbital floor was reconstructed with a pre-fabricated titanium meshwork scaffold, and an immediate obturator and dental prosthesis was inserted (Fig 9). Histological examination of the resected maxilla revealed the presence of residual tumor at the posterior margin, for which the patient had to undergo surgical removal of the pterygoid plates and additional chemotherapy. 


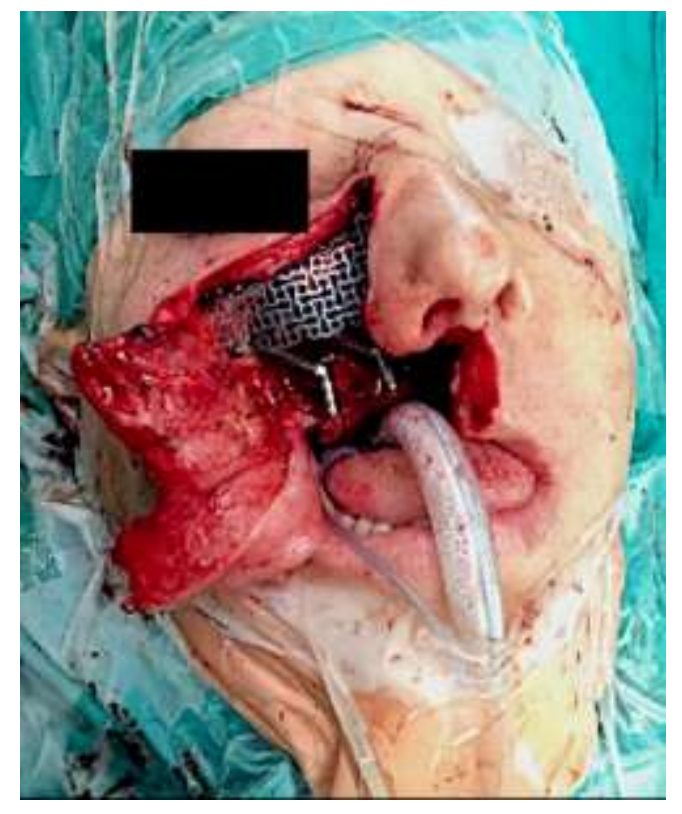

Figure 9 Right subtotal maxillectomy with two oncology implants emerging from the right zygoma and meshwork scaffold supporting the right orbital floor.

Subsequent healing and recovery were uneventful, and a final overdenture/obturator on an implant-retained titanium bar were fabricated to restore the patient to full speech and masticatory function, three months after tumor ablative surgery (Fig 10).

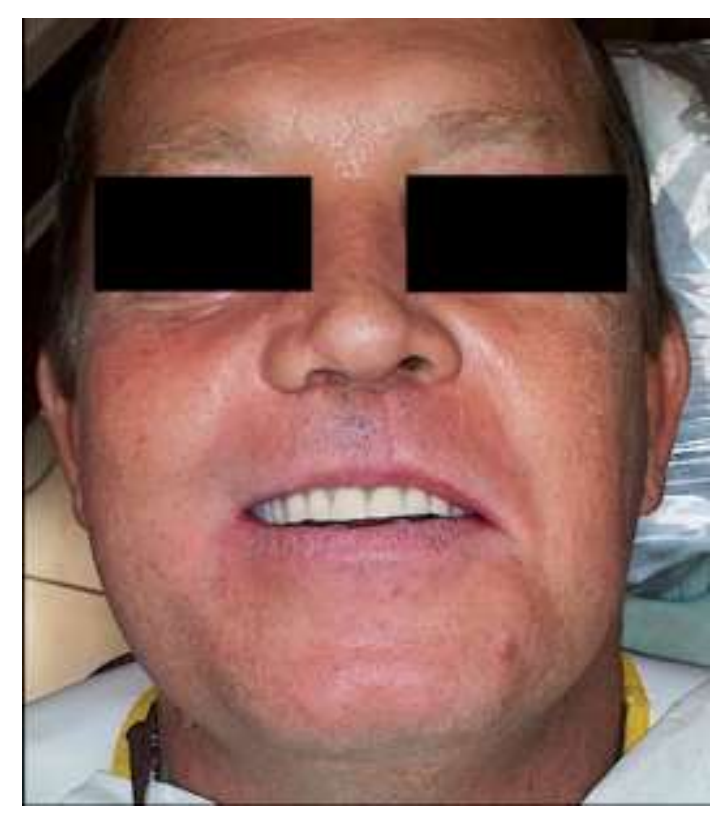

Figure 10 Post-operative frontal view of the patient with final prosthesis in place. 


\section{Case 4}

A 73-year-old female patient presented with a diagnosed squamous cell carcinoma involving the right maxilla, alveolus, palate and buccal mucosa. CT and magnetic resonance imaging (MRI) scans revealed extensive tumor infiltration into the right maxillary sinus and the nasal septum. As before, these scans were used to produce 3D models of the patient's maxilla and mandible, to assist in pre-operative surgical planning, and dental prosthesis/obturator fabrication. Surgical removal involved a right subtotal maxillectomy, during which time two zygomatic implants were placed into the right zygoma. These implants retained the immediate obturator and dental prosthesis (Figs 11 and 12) securely via titanium screws. The patient then underwent a course of post-operative radiotherapy. Recovery was uneventful and a definitive prosthesis was manufactured and inserted six months later.

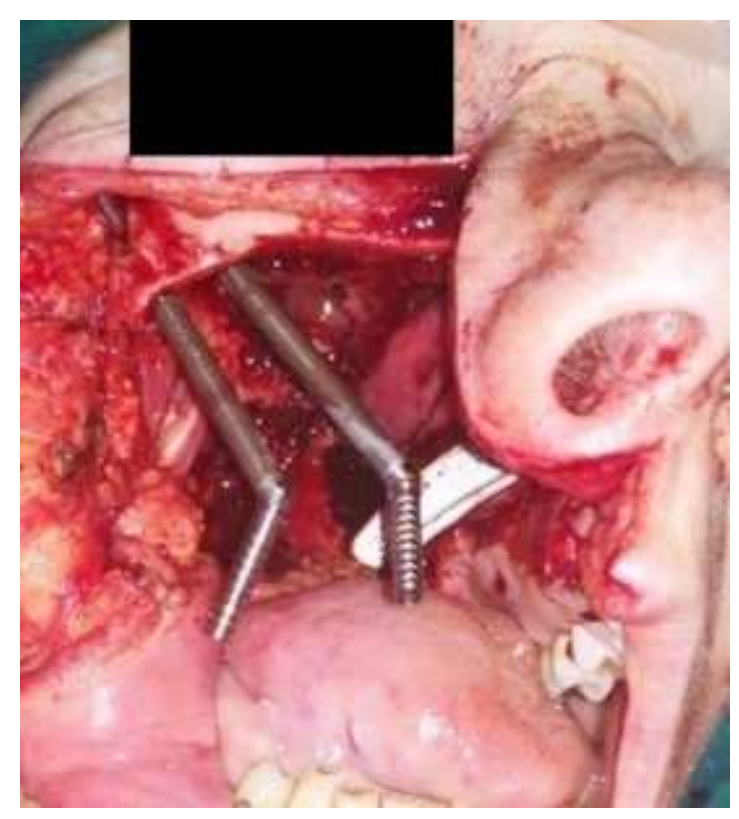

Figure 11 Right subtotal maxillectomy with two zygomatic implants to retain an obturator and dental prosthesis. 


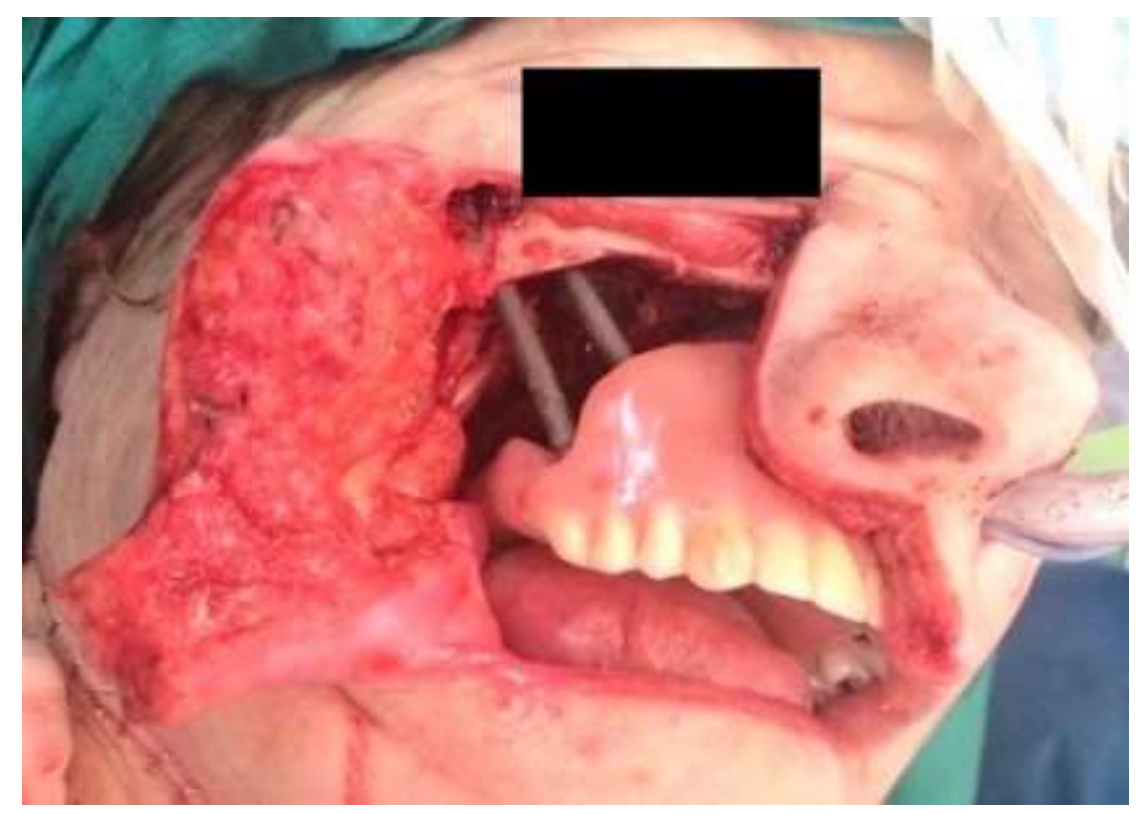

Figure 12 Dental prosthesis fixed to two zygomatic implants to obturate the maxillectomy defect and restore oral function.

\section{Discussion}

Surgical resection of oral and midfacial tumors often leads to considerable facial disfigurement and oral dysfunction, with potentially major psychosocial implications. ${ }^{9}$ Advances in technology along with increased interdisciplinary collaboration have made it possible to restore function and esthetics to these patients immediately, or very soon after devastating surgery. This greatly improves their post-operative recovery time and quality of life.

It has been advised that when using zygomatic implants to support prostheses in large maxillary defects, these should be allowed a 4-6-month period for osseintegration prior to loading. ${ }^{6}$ Studies have shown, however, that even with this delay, there is a $25 \%$ implant failure rate. ${ }^{10}$ Interestingly, all these failures occurred before loading, suggesting that other factors could be implicated in their failure. The 
main non-mechanical contributor to this failure was radiation therapy in these patients. ${ }^{10}$

Studies have also shown that placement of implants, before radiotherapy, at the time of the tumor ablative surgery, greatly improves their chance of survival. ${ }^{11,12}$ Benefits of this are that osseointegration can take place prior to the harmful effects of radiotherapy, and to avoid further surgery at a later stage to aid the oro-facial rehabilitation. ${ }^{11}$ Based on these results, it has been decided by our multi-disciplinary team, to immediately place zygomatic implants at the time of resective surgeries, and prior to radiotherapy commencement. These implants can achieve primary bicortical stability even when large parts of the maxilla have been resected, and may be the only option for stabilizing an obturator prosthesis. ${ }^{16}$ Their immediate placement combined with immediate provisionalization with obturators, dental, and facial prostheses has been shown to be advantageous in improving post-operative functional and psychological well-being and recovery in these patients. These patients are no longer burdened by the additional difficulties of eating, communicating and socializing, while at the same time trying to come to terms with their devastating and life changing surgery. Though there are several studies reporting on the success of these implants in restoring maxillectomy defects, ${ }^{17-19}$ very few ${ }^{9}$ have reported on their immediate placement at the time of resection with immediate obturation.

To the best of the authors' knowledge, there are no reports in the current literature regarding immediate provisionalization in patients with mid-facial defects following maxillary tumor ablative surgery. Although this treatment modality promises to be a 
viable and valuable option in selected patients, it is reliant on close co-operation of all members of the multi-disciplinary team, along with careful and meticulous surgical and prosthodontic planning.

For most cases involving maxillary tumor resection, the authors suggest the following treatment protocol:

- Pre-operative CT scans of the head and neck region (incorporating tumor sites and surrounding tissues), as well as facial scans, if possible

- 3D model production (via rapid prototyping/3D printing)

- Multi-disciplinary pre-surgical planning and model surgery to establish extent of resections, and positioning of zygomatic implants

- Duplication of resected models into plaster casts

- Fabrication of dental/obturator, and facial prostheses on duplicate casts

- Intra-operative tumor resection, with immediate zygomatic implant placement

- Intra-operative modification of dental/obturator prostheses, so that these are retained and supported by the zygomatic implants (prostheses are connected to the implants by titanium cylinders, screws, and self-curing relining materials)

- Sealing residual defects with BIPP gauze

Patients should be monitored on a regular basis (monthly), and final prostheses should be fabricated within 3-6 months following surgery. The use of zygomatic implants allows for constant surveillance of the resected site, and facial prostheses will usually require replacement every $1.5-2$ years. $^{13}$ 


\section{Conclusion}

The placement of zygomatic implants at the time of tumor ablative surgery to aid in the retention of oral and facial prostheses has been found to be a viable treatment option. Loading the implants and immediately placing an interim prosthesis at the time of tumor resection, greatly improves post-operative patient recovery and comfort, as it improves patients' ability to cope with often devastating facial and functional defects. Due to the aggressive nature of oro-facial tumors, most patients require post-operative radiotherapy. This is known to be associated with harmful effects on hard and soft tissues, that can affect healing and osseointegration. The added advantage of placing implants at the time of tumor resection, is that osseintegration can occur before radiotherapy is initiated.

\section{References}

1. Santamaria E, Cordeiro PG. Reconstruction of maxillectomy and midfacial defects with free tissue transfer. J Surg Oncol. 2006; 94:522-31.

2. Fernandes N, van den Heever J, Hoogendijk C, Botha S, Booysen G, Els J. Reconstruction of an Extensive Midfacial Defect Using Additive Manufacturing Techniques. J Prosthodont. 2016; 25(7):589-94.

3. O'Connell DA, Futran ND. Reconstruction of the midface and maxilla. Curr Opin Otolaryngol Head Neck Surg. 2010; 18(4):304-10.

4. Sinn DP, Bedrossian E, Vest AK. Craniofacial implant surgery. Oral Maxillofac Surg Clin North Am. 2011; 23(2):321-35.

5. Toso SM, Menzel K, Motzkus Y, et al. Anaplastology in times of facial transplantation: Still a reasonable treatment option? J Craniomaxillofac Surg. 2015; 43(7):1049-53. 
6. Trevisiol L, Procacci P, D'Agostino A, Ferrari F, De Santis D, Nocini PF. Rehabilitation of a complex midfacial defect by means of a zygoma-implantsupported prosthesis and nasal epithesis: a novel technique. Int J Implant Dent. $2016 ; 2(1): 7$.

7. Malevez C. The zygomatic implant for the totally atrophied maxilla. Oral Maxillofac Surg. 2012; 27:439-50.

8. Schmidt B, Pogrel MA, Young CW, Sharma AB. Reconstruction of extensive maxillary defects using zygomaticus implants. J Oral Maxillofac Surg. 2004; 62:82-9.

9. Boyes-Varly JG, Howes DG, Davidge-Pitts KD, Branemark P-I, McAlpine JA. A protocol for maxillary reconstruction following oncology resection using zygomatic implants. Int J Prosthodont. 2007; 20:521-31.

10. Leonardi A, Buonaccorsi S, Pellacchia V, Moricca LM, Indrizzi E, Fini G. Maxillofacial prosthetic rehabilitation using extraoral implants. J Craniofac Surg. 2008; 19:398-405.

11. Robb GL, Marunick MT, Martin JW, Zlotolow IM. Midface reconstruction: surgical reconstruction versus prosthesis. Head Neck. 2001; 23(1):48-58.

12. Runyan CM, Sharma V, Staffenberg DA, et al. Jaw in a day: state of the art in maxillary reconstruction. J Craniofac Surg. 2016; 27(8):2101-4.

13. Visser A, Raghoebar GM, van Oort RP, Vissink A. Fate of implant-retained craniofacial prostheses: lifespan and aftercare. Int J Oral Maxillofac Implants. 2008; 23(1):89-98.

14. Cordeiro PG, Chen CM. A 15-year review of midface reconstruction after total and subtotal maxillectomy: part I. Algorithm and outcomes. Plast Reconstr Surg. $2012 ; 129(1): 124-36$. 
15. Crossland GJ, Bath AP. Bismuth iodoform paraffin paste: a review. J Laryngol Otol. $2011 ; 125(9): 891-5$.

16. D'Agostino A, Pasquale P, Ferrari F, Trevisiol L, Francesco NP. Zygoma implantsupported prosthetic rehabilitation of a patient after subtotal bilateral maxillectomy. J Craniofac Surg. 2013; 24(2):e159-62.

17. Parel SM, Branemark P-I, Ohmell LO, Svensson B. Remote implant anchorage for the rehabilitation of maxillary defects. J Prosthet Dent. 2001; 86(4):377-81.

18. Shirota T, Shimodaira O, Matsui Y, Hatori M, Shintani S. Zygoma implantsupported prosthetic rehabilitation of a patient with a maxillary defect. Int J Oral Maxillofac Surg. 2011; 40(1):113-7.

19. Bowden JR, Flood TR, Downie IP. Zygomaticus implants for retention of nasal prostheses after rhinectomy. Br J Oral Maxillofac Surg. 2006; 44(1):54-61. 